Portland State University

PDXScholar

Electrical and Computer Engineering Faculty

Publications and Presentations

Electrical and Computer Engineering

9-1-1999

\title{
Double-Pass High-Gain Laser Amplifiers
}

Janet M. Casperson

Whitman College

Frederick G. Moore

Whitman College

Lee W. Casperson

Portland State University

Follow this and additional works at: https://pdxscholar.library.pdx.edu/ece_fac

Part of the Electrical and Computer Engineering Commons

Let us know how access to this document benefits you.

\section{Citation Details}

Casperson, J. M., Moore, F. G., \& Casperson, L. W. (1999). Double-pass high-gain laser amplifiers. Journal of Applied Physics, 86(6), 2967-2973.

This Article is brought to you for free and open access. It has been accepted for inclusion in Electrical and Computer Engineering Faculty Publications and Presentations by an authorized administrator of PDXScholar. Please contact us if we can make this document more accessible: pdxscholar@pdx.edu. 


\title{
Double-pass high-gain laser amplifiers
}

\author{
Janet M. Casperson ${ }^{\text {a) }}$ and Frederick G. Moore \\ Department of Physics, Whitman College, 345 Boyer Avenue, Walla Walla, Washington 99362-2083 \\ Lee W. Casperson ${ }^{\text {b) }}$ \\ Departments of Physics and Electrical and Computer Engineering, Portland State University, P.O. Box \\ 751, Portland, Oregon 97207-0751
}

(Received 3 May 1999; accepted for publication 9 June 1999)

\begin{abstract}
Double-pass laser amplifiers have advantages of compactness and efficiency in the amplification of optical signals, and such amplifiers have been employed in a wide range of optical systems. Work in this area is reviewed briefly, and analytical solutions are obtained for the intensity of the electromagnetic waves in double-pass homogeneously-broadened high-gain laser amplifiers. Expressions are derived relating the output power to the input, including the effects of arbitrary mirror reflectivity and frequency detuning from line center. In the limits of weak saturation and of high reflectivity the results are consistent with earlier studies. (C) 1999 American Institute of
\end{abstract} Physics. [S0021-8979(99)05318-9]

\section{INTRODUCTION}

In recent years it has been recognized that the doublepass laser amplifier configuration can have significant advantages over more basic single-pass amplifier designs. For example, with negligible saturation, signals in an amplifier grow exponentially with distance; and thus, a double-pass arrangement leads, in effect, to a squaring of the small signal gain. Also, with a saturating output the double-pass configuration may make more efficient use of the gain region near the amplifier input. For these reasons of gain, compactness, and efficiency, the double-pass amplifier has been attracting increased attention. Improvements in related optical components such as circulators, reflectors, antireflection coatings, and polarization rotators have made the construction of highquality double-pass amplifiers a more straightforward task. The wide-ranging applications of double-pass amplifiers and the models that might describe them seem not to have been reviewed previously. A brief guide to some of the research on this topic is included in the following paragraphs.

The advantages of double-pass amplification have long been recognized, and the earliest applications involved superfluorescent systems. When the single-pass gain of a laser amplifier is sufficiently large, spontaneously emitted photons from one end of the amplifier are strongly amplified before reaching the other end. The resulting superfluorescent intensity spectrum may be much narrower and more intense than the spectrum of the unamplified spontaneous emission. ${ }^{1,2}$ Typically, one end of such an amplifier is made highly reflecting to increase the amplifier output, while the other end may be antireflection coated to prevent the onset of laser oscillation. Analytical models for the behavior of such systems have been developed. ${ }^{3,4}$ One application of superfluo-

\footnotetext{
a) During part of this work the author was at the LIGO Hanford Observatory, P.O. Box 1970, Richland, WA 99352-0539.

b)Electronic mail: 1caspers@ee.pdx.edu
}

rescent lasers is as an absolute wavelength standard. Lasers of this type have also been employed as pump sources for other lasers, and for applications such as fluorescence and phosphorescence spectroscopy, charge carrier lifetime measurements in semiconductors, and calibration of high-speed photodetectors. $^{5}$

Double-pass amplifiers also are being used to enhance an externally introduced optical signal. In this case the input and output signals are both present at the same end of the amplifier, and techniques for separating them were reported as early as 1972. ${ }^{6}$ Advantages of double-pass amplification for gas lasers have long been recognized, and applications to waveguide ${ }^{7}$ and nonwaveguide gas lasers have also been developed. ${ }^{8}$ Improved transverse intensity profiles in doublepass gas lasers have been obtained by incorporation of phase conjugate mirrors. ${ }^{9-11}$

Applications of double-pass amplifiers to fiber waveguide laser systems have been an area of substantial recent interest. Some double-pass fiber amplifiers have separated the input and output beams by using an input polarizing beam splitter together with a Faraday rotator mirror, ${ }^{12}$ while others have employed an input/output optical circulator. ${ }^{13-21}$ In the circulator case the signal feedback reflector can be either an ordinary mirror or coating, ${ }^{13-17,20}$ or a more complicated reflector structure that might, for example, provide gain stabilization and equalization. ${ }^{18,19,21}$ Sometimes only the pump beam is double passed through the fiber amplifier. ${ }^{22}$ Waveguide double-pass amplifiers based on $\mathrm{Er}: \mathrm{LiNbO}_{3}{ }^{23-25}$ and $\mathrm{Nd}: \mathrm{Y}_{3} \mathrm{Al}_{5} \mathrm{O}_{12}$ have also been developed. ${ }^{26}$ Nonwaveguide double-pass YAG laser amplifiers have been investigated for intersatellite communication ${ }^{27}$ and gravitational wave detection, ${ }^{28}$ and a double-pass Nd:YAG laser with a phase conjugate mirror has been demonstrated. ${ }^{29}$

Some of the leading application areas for double-pass lasers involve semiconductor diode lasers. ${ }^{30-35}$ Some of these lasers use circulators to separate the input and output beams, ${ }^{31}$ while others are based on angular separation. ${ }^{32,33,35}$ 
The possibility of improved beam quality in double-pass semiconductor laser amplifiers with phase conjugate mirrors has also been considered. ${ }^{30}$

In spite of the growing range of applications for doublepass laser amplifiers, most studies are lacking a detailed and rigorous underlying model. There have only been more limited efforts to obtain analytical or graphical guidelines to aid in the design of these systems. A one-dimensional analytical model for a double-pass amplifier having a high-reflectivity end mirror was developed and applied to a $\mathrm{CO}_{2}$ laser system. $^{7}$ A similar model was formulated to include in an approximate way some of the effects of distributed losses and amplified spontaneous emission. ${ }^{36}$ Most other treatments have been more specialized and dependent on numerical methods. In one analysis the amplifying medium is allowed to move transversely to the spatially varying laser beams, while the reflection coefficient is intensity dependent. ${ }^{37} \mathrm{Ap}$ proximate analytical models for two-level amplifiers have been developed for use with certain fiber systems. ${ }^{38,39}$ Others concern transverse field variations in double-pass lasers with phase-conjugating mirrors. ${ }^{9-11}$ Double-pass waveguide amplifiers have been studied numerically, including $\mathrm{Er}^{3+}$-doped $\mathrm{LiNbO}_{3}$ and $\mathrm{Nd}^{3+}$-doped silica waveguides. ${ }^{23,25}$ Double-pass amplification has also been studied numerically in a variety of semiconductor diode laser configurations. Thus, transverse effects and filamenting have been considered in semiconductor lasers in which the right and left travelling components are competing for the same gain medium. ${ }^{30,34}$ In more complicated broad-area double-pass semiconductor lasers, the input and output beams are nonparallel to reduce filamentation and to simplify the physical separation of the input and output beams, but detailed modeling of such systems necessarily relies on numerical methods. ${ }^{32}$ While there are important benefits in developing the most rigorous multidimensional models for all lasers of practical interest, there is also value in retaining simpler semi-analytic models for use in more basic laser designs and as first estimates for the behavior of more complex laser structures.
In this study we develop a model for the behavior of a double-pass laser amplifier that consists of a general homogeneously broadened amplifying medium and a single feedback mirror. We do not make the average intensity approximation, and thus, we allow for arbitrarily high gain values per pass and arbitrary reflectivities. In the limits of weak saturation and of high reflectivity the results are consistent with earlier studies. If the gain coefficient is considered to be negative, the results are also applicable to absorbing media and thus may be applied to amplifiers having double-pass pump configurations.

Another subject discussed briefly concerns the phase of the fields inside the active medium. Most amplifier models include only intensities, neglecting the phase. The amplifier model we employ also includes the effects of frequency detunings from the gain center frequency as well as saturationdependent dispersion. Thus, in principle, the analysis can be generalized to include coherent reflections from a possible reflector at the input end or the incorporation of the amplifier in some other interferometric system.

The basic equations for the electromagnetic fields in a saturating homogeneously-broadened amplifying medium are developed in Sec. II. The equations are integrated in Sec. III, and they are shown to reduce to previously obtained results in the limits of weak saturation or high reflectivity. Detailed solutions for the general double-pass amplifier configuration are reported in Sec. IV.

\section{FIELD AND INTENSITY EQUATIONS}

The starting point for any rigorous treatment of the interaction of light with atoms is the Maxwell and Schrödinger equations. Starting from these equations one can develop a semiclassical model for the propagation of optical signals in a laser amplifier. A double-pass amplifier is necessarily bidirectional, and for brevity we simply adopt the known form for the plane-wave field equations in a bidirectional homogeneously broadened laser amplifier. These equations can be written $^{40}$

$$
\begin{aligned}
& \frac{d A^{+}(z)}{d z}=-\eta\left\{A^{+}(z)-\frac{(1+i y) D_{0}(z) A^{+}(z)}{1+y^{2}+\left[\left|A^{+}(z)\right|^{2}+\left|A^{-}(z)\right|^{2}+2\left|A^{+}(z) A^{-}(z)\right| \cos (2 k z)\right]}\right\}, \\
& \frac{d A^{-}(z)}{d z}=+\eta\left\{A^{-}(z)-\frac{(1+i y) D_{0}(z) A^{-}(z)}{1+y^{2}+\left[\left|A^{+}(z)\right|^{2}+\left|A^{-}(z)\right|^{2}+2\left|A^{+}(z) A^{-}(z)\right| \cos (2 k z)\right]}\right\},
\end{aligned}
$$

where $A^{+}(z)$ and $A^{-}(z)$ are the normalized complex amplitudes of the right and left traveling waves at a point $z$ inside the amplifier, the coefficient $\eta$ corresponds to a loss rate per unit of propagation distance, $D_{0}(z)$ is the normalized unsaturated population difference, and the normalized optical frequency is $y=\left(\omega-\omega_{0}\right) / \gamma$ with $\omega_{0}$ the center frequency of the transition and $\gamma$ the decay rate for the off-diagonal elements of the density matrix. The field amplitudes have been normalized in such a way that in a one-dimensional amplifier $\left[A^{+}(z) \neq 0, A^{-}(z)=0\right]$ tuned to line center $(y=0)$ the field gain is reduced to half of its unsaturated value $\left[\eta D_{0}(z)\right]$ when the magnitude of the field is equal to unity $\left[\left|A^{+}(z)\right|=1\right]$. The cosine term in the saturation denominator results from interference between the right and left travelingwave field components. The relative phases of the right and left waves have been assumed to be unimportant, but they could be included by shifting the arguments of the cosine functions. These equations account for the effects of line center detuning, longitudinal hole burning, and the possibility of high gain per pass. 
The earlier equations govern the spatial evolution of the electromagnetic fields. It is also useful to convert them to an equivalent set of equations for the right and left traveling intensities. By defining normalized intensities $s I^{+}(z)$ $=\left|A^{+}(z)\right|^{2}$ and $s I^{-}=\left|A^{-}(z)\right|^{2}$, one can obtain from Eqs. (1) and (2)

$$
\begin{aligned}
& \frac{d I^{+}(z)}{d z}=-2 \eta\left(I^{+}(z)-\frac{D_{0}(z) I^{+}(z)}{1+y^{2}+s\left\{I^{+}(z)+I^{-}(z)+2\left[I^{+}(z) I^{-}(z)\right]^{1 / 2} \cos (2 k z)\right\}}\right), \\
& \frac{d I^{-}(z)}{d z}=+2 \eta\left(I^{-}(z)-\frac{D_{0}(z) I^{-}(z)}{1+y^{2}+s\left\{I^{+}(z)+I^{-}(z)+2\left[I^{+}(z) I^{-}(z)\right]^{1 / 2} \cos (2 k z)\right\}}\right) .
\end{aligned}
$$

In this notation $I^{+}$and $I^{-}$are the actual intensities and $s$ is a saturation parameter. It should be noticed that although the set of Eqs. (1) and (2) closely resemble Eqs. (3) and (4), in going from the former to the latter information on the phase of the waves has been lost.

It follows from Eqs. (1) and (2) that the product of the right and left field amplitudes is equal to a constant. This result can be written $A^{+} A^{-}=$constant $\equiv s a$ and $\left|A^{+}\right|{ }^{2}\left|A^{-}\right|^{2}$ $=s^{2} I^{+} I^{-}=s^{2}|a|^{2}$, where the constant $a$ has been introduced.
The complex electric field amplitudes now can be written in terms of only one variable in the form

$$
\begin{aligned}
& A^{+}(z)=s^{1 / 2} a^{1 / 2} \exp [(1+i y) \alpha(z)+i y \eta z+i \phi], \\
& A^{-}(z)=s^{1 / 2} a^{1 / 2} \exp [-(1+i y) \alpha(z)-i y \eta z-i \phi],
\end{aligned}
$$

where $\alpha(z)$ is a (real) gain coefficient for the field governed by

$$
\frac{d \alpha(z)}{d z}=-\eta\left(1-\frac{D_{0}(z)}{1+y^{2}+s|a|\{\exp [2 \alpha(z)]+\exp [-2 \alpha(z)]+2 \cos (2 k z)\}}\right),
$$

and $\phi$ is an integration constant. Thus, the original coupled complex differential equations have been reduced to a single real equation. It would still be necessary to integrate Eq. (7) and to obtain the constant $a$ using boundary conditions for the double-pass amplifier.

Since the absolute phase of the reflected wave would usually not be of interest, it may be more convenient to work initially with the intensity equations rather than with Eq. (7). It follows from Eqs. (5) and (6) that the intensities can be written

$$
\begin{aligned}
& s I^{+}(z)=\left|A^{+}(z)\right|^{2}=s|a| \exp [2 \alpha(z)], \\
& s I^{-}(z)=\left|A^{-}(z)\right|^{2}=s|a| \exp [-2 \alpha(z)] .
\end{aligned}
$$

Thus, the function $\alpha(z)$ needed in Eqs. (5) and (6) can always be obtained from the intensities. To facilitate the actual integrations some further simplifications can usually be employed.

In many practical cases, the effects of the longitudinal spatial hole burning can be neglected by arguing that the oscillating term $\cos (2 k z)$ in the denominator of Eqs. (3) and (4) is significant only in the short distance within a high-gain amplifier where the magnitudes of the right and left waves are about the same. Alternatively, one may often argue that spatial cross relaxation causes this term to average to zero. ${ }^{41}$ Another common simplification is the neglect of the distributed loss term, the first term on the right-hand side of Eqs.
(3) and (4). With these two approximations and the product result mentioned earlier, Eq. (3) can be written

$$
\frac{d I^{+}(z)}{d z}=\eta \frac{2 D_{0}(z) I^{+}(z)}{1+y^{2}+s\left[I^{+}(z)+|a|^{2} / I^{+}(z)\right]},
$$

where the relationship preceding Eq. (5) has been used. We will also assume that the unsaturated population difference $D_{0}$ is independent of position $z$, and thus Eq. (10) is now in a familiar integrable form. The corresponding phase information can readily be obtained by application of Eqs. (5) and (6) once the intensity has been solved for.

\section{SOLUTION OF THE WAVE EQUATIONS}

It was shown earlier that useful information about double-pass lasers can be obtained from an integration of the intensity wave equations. By introducing the new gain and saturation parameters

$$
\begin{aligned}
& g=\eta \frac{2 D_{0}}{1+y^{2}}, \\
& s^{\prime}=\frac{s}{1+y^{2}},
\end{aligned}
$$

Eq. (10) can be written in the more compact form

$$
\frac{d I^{+}(z)}{d z}=\frac{g I^{+}(z)}{1+s^{\prime}\left[I^{+}(z)+|a|^{2} / I^{+}(z)\right]} .
$$




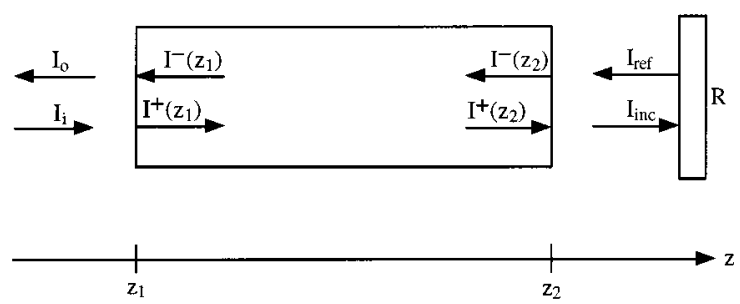

FIG. 1. Definitions of intensities in a double-pass laser amplifier.

This equation can be integrated and the result is

$s^{\prime}\left[I^{+}\left(z_{2}\right)-I^{+}\left(z_{1}\right)\right]+\ln \left[\frac{I^{+}\left(z_{2}\right)}{I^{+}\left(z_{1}\right)}\right]-\frac{s^{\prime}|a|^{2}}{I^{+}\left(z_{2}\right)}+\frac{s^{\prime}|a|^{2}}{I^{+}\left(z_{1}\right)}=g L$.

The position and direction notation employed here is indicated in Fig. 1. This figure shows a double-pass amplifier in which the input signal comes from the left and the reflector at the right-hand end has a reflection coefficient of $R$.

Equation (14) relates the positive traveling intensity at the two ends of the amplifier, the gain-length product $g L$, and the modulus of the parameter $a$. On the other hand, the quantities $I^{+}\left(z_{1}\right), I^{+}\left(z_{2}\right)$, and $a$ can be related to the amplifier input and output via appropriate boundary conditions. As noted earlier, the product of the right and left intensities is independent of the position in the amplifier, and at the two ends of the laser this relationship implies

$$
I^{+}\left(z_{1}\right) I^{-}\left(z_{1}\right)=I^{+}\left(z_{2}\right) I^{-}\left(z_{2}\right)=|a|^{2} .
$$

On the other hand, the intensities at the right-hand end are also related to the intensity reflection coefficient $R$ according to

$$
I^{-}\left(z_{2}\right)=R I^{+}\left(z_{2}\right) .
$$

From Eqs. (15) and (16) the constant $|a|^{2}$ is

$$
|a|^{2}=R\left[I^{+}\left(z_{2}\right)\right]^{2},
$$

and with these substitutions $|a|^{2}$ can be eliminated from Eq. (14) yielding

$$
\begin{gathered}
s^{\prime}\left[(1-R) I^{+}\left(z_{2}\right)+I^{-}\left(z_{1}\right)-I^{+}\left(z_{1}\right)\right] \\
+\ln \left[I^{+}\left(z_{2}\right) / I^{+}\left(z_{1}\right)\right]=g L .
\end{gathered}
$$

The intensity $I^{+}\left(z_{2}\right)$ can also be eliminated from Eq. (18) by means of the boundary conditions. Thus, Eqs. (15) and (17) may first be combined to obtain the relationship

$$
I^{+}\left(z_{2}\right)=\left[I^{+}\left(z_{1}\right) I^{-}\left(z_{1}\right) / R\right]^{1 / 2} .
$$

When Eq. (19) is substituted into Eq. (18), the result is

$$
\begin{aligned}
2 s^{\prime}\{ & (1-R)\left[I^{+}\left(z_{1}\right) I^{-}\left(z_{1}\right) / R\right]^{1 / 2}+I^{-}\left(z_{1}\right) \\
& \left.-I^{+}\left(z_{1}\right)\right\}+\ln \left\{I^{-}\left(z_{1}\right) /\left[R I^{+}\left(z_{1}\right)\right]\right\}=2 g l .
\end{aligned}
$$

In a double-pass amplifier having one mirror, the intensities $I^{+}\left(z_{1}\right)$ and $I^{-}\left(z_{1}\right)$ can be replaced by the input intensity $I_{i}$ and the output intensity $I_{0}$, respectively. Thus, Eq. (20) can also be written

$$
2 s^{\prime}\left[(1-R)\left(I_{i} I_{0} / R\right)^{1 / 2}+I_{0}-I_{i}\right]+\ln \left[I_{0} /\left(R I_{i}\right)\right]=2 g l .
$$

Equation (21) is a closed form implicit analytic formula relating the input and output intensities. This equation simplifies a little if $I_{i}$ and $I_{0}$ are replaced by the normalized intensities $J_{i}=s^{\prime} I_{i}$ and $J_{0}=s^{\prime} I_{0}$, and the result of these substitutions is

$$
2\left[(1-R)\left(J_{i} J_{0} / R\right)^{1 / 2}+J_{0}-J_{i}\right]+\ln \left[J_{0} /\left(R J_{i}\right)\right]=2 g L .
$$

It may be noted that this relationship reduces to known results in the appropriate limits. For example, if the intensity is very small the logarithmic terms dominate, and Eq. (22) can be written in the form

$$
J_{0}=J_{i} R \exp (2 g L) .
$$

This is the familiar exponential behavior that one would expect for two passes through an unsaturated amplifier. If the reflector has a very high reflection coefficient $(R \approx 1)$, Eq. (22) is approximately

$$
2\left(J_{0}-J_{i}\right)+\ln \left(J_{0} / J_{i}\right)=2 g L,
$$

and an analogous result has been obtained previously. ${ }^{7}$

As a final analytic example, it may be noted that, in the limit that the intensity is very large, the logarithmic terms may sometimes become unimportant. In this case Eq. (22) can be written in the form

$$
(1-R)\left(J_{i} J_{0} / R\right)^{1 / 2}+J_{0}-J_{i}=g L .
$$

This can be recognized as a quadratic equation for the output intensity, and thus, it can be solved explicitly. The result is

$J_{0}=\left\{\frac{-(1-R)\left(J_{i} / R\right)^{1 / 2}+\left[(1-R)^{2} J_{i} / R+4\left(J_{i}+g L\right)\right]^{1 / 2}}{2}\right\}^{2}$.

Equation (26) describes the behavior of a saturated double-pass laser amplifier having arbitrary mirror reflectivity. If the reflection coefficient $R$ is close to unity $(R \approx 1)$, this output formula simplifies to

$$
J_{0}=J_{i}+g L \text {. }
$$

With strong saturation and high reflectivity the signal sweeps out all available population inversion, and with no loss at the mirror the output intensity increases linearly with the amplifier length.

\section{RESULTS}

Equation (22) is an implicit analytic relationship between the input and output intensities of a double-pass laser amplifier. Solutions to this equation may be readily obtained by means of Newton's rule or some other iterative procedure. Figure 2 includes plots of the output intensity as a function of the input intensity for the mirror reflection coefficient $R=0.3$ and various values of the gain-length product $g L$. This intermediate value of reflectivity would be appropriate for some uncoated semiconductor laser surfaces. In Fig. 2(a) is a plot of the output for input values from 0.0 to 1.0, and also included in the plot are dashed lines representing the linear unsaturated input-output relationship given in Eq. (23). In Fig. 2(b) are the same plots for input values from 0.0 to 10.0 together with dashed lines representing the saturated 

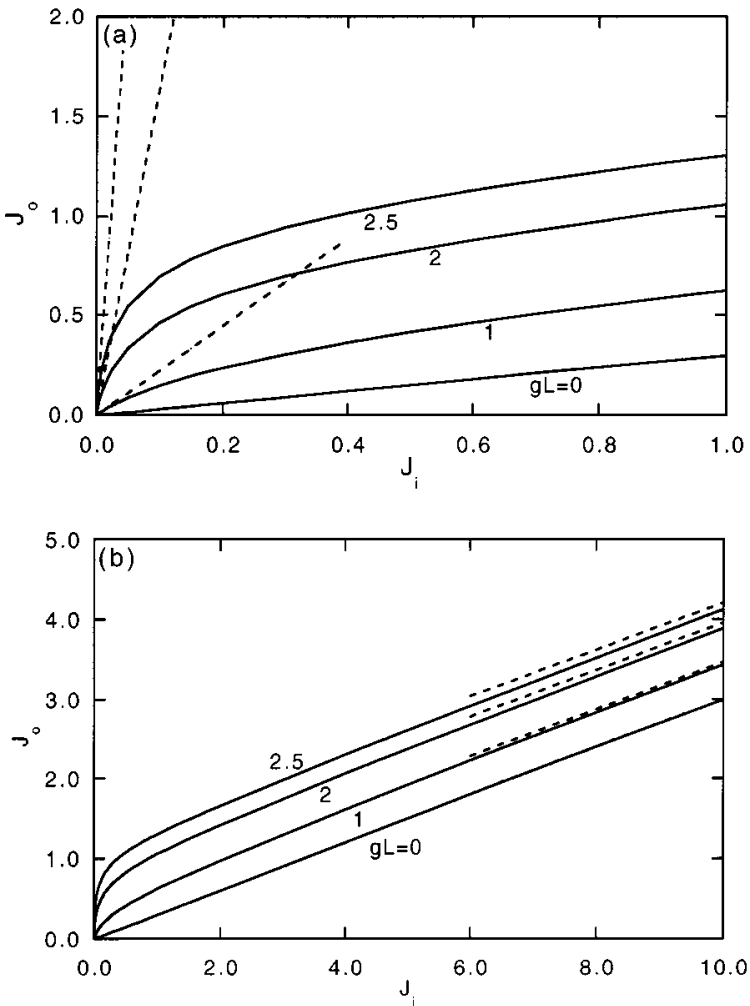

FIG. 2. Normalized output intensity as a function of input for various values of the gain-length product $g L$ in a double-pass laser amplifier having the mirror reflection coefficient $R=0.3$. In (a) the dashed lines represent the linear unsaturated input-output relationship given in Eq. (23), and in (b) the dashed lines represent the saturated input-output relationship given in Eq. (26).

input-output relationship given in Eq. (26). It is evident from these plots that the output intensity initially rises rapidly with input and then more slowly and almost linearly after the amplifier is highly saturated.

It is reasonable to suppose that a higher reflection coefficient than that used in calculating the results shown in Fig. 2 would be desirable and readily obtainable for double-pass amplifier systems. For instance the reflection of a semiconductor surface could be increased in a practical double-pass system by appropriate reflective coatings. However, high reflectivities have not been obtainable in many of the previous double-pass amplifier studies due to the use of more complex waveguide and reflector techniques. In fiber-optic systems, for example, reported reflection coefficients are sometimes in the range of $0.4-0.6,{ }^{15}$ and wide ranges of values also occur with waveguide amplifier configurations ${ }^{25}$ or phaseconjugate reflectors. ${ }^{10}$ Thus, there is value in being able to calculate amplifier performance for arbitrary mirror properties.

Figure 3 includes plots of the output intensity as a function of the input intensity for the intermediate gain-length product $g L=2.5$ and various values of the reflection coefficient $R$. With $R=1$ the results also follow from the simpler Eq. (24). In Fig. 3(a) is a plot of the output for input values from 0.0 to 1.0, and also included in the plot are dashed lines representing the linear unsaturated input-output relationship given in Eq. (23). In Fig. 3(b) are the same plots for input
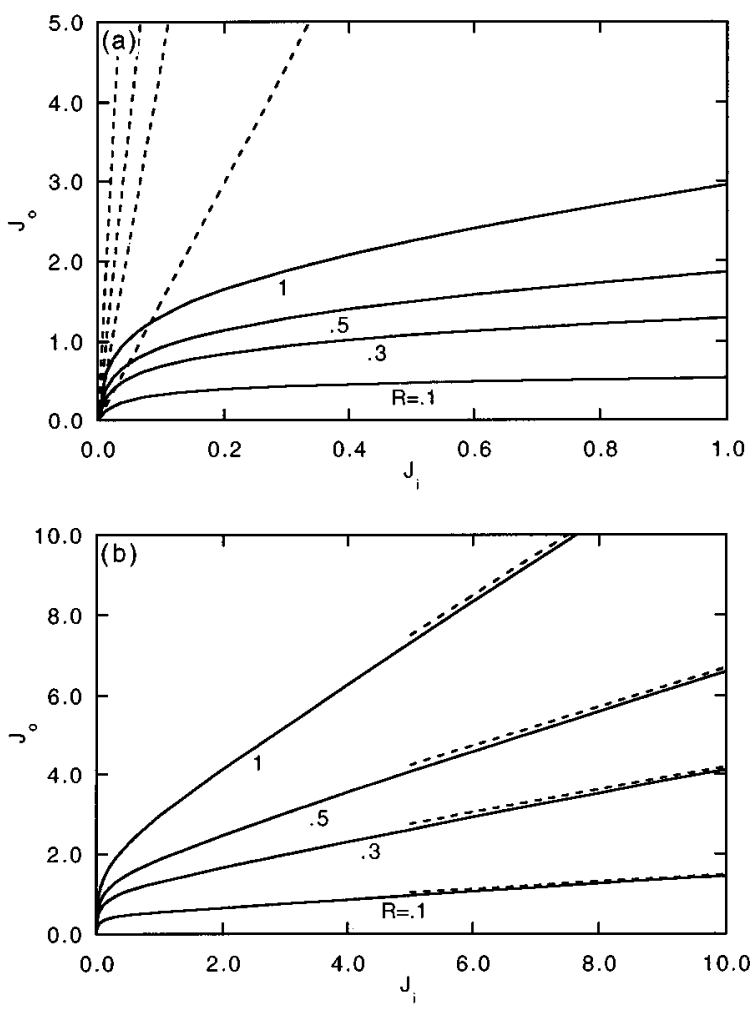

FIG. 3. Normalized output intensity as a function of input for various values of the mirror reflection coefficient $R$ in a double-pass laser amplifier having the gain-length product $g L=2.5$. In (a) the dashed lines represent the linear unsaturated input-output relationship given in Eq. (23), and in (b) the dashed lines represent the saturated input-output relationship given in Eq. (26).

values from 0.0 to 10.0 together with dashed lines representing the saturated input-output relationship given in Eq. (26). As in Fig. 2, the output intensity initially rises rapidly with input and then more slowly and almost linearly after the amplifier is highly saturated.

In Fig. 4 is a set of plots of the output $J_{0}$ versus $g L$ for various values of the input $J_{i}$. It is clear from these plots that for some gain-length products $g L$, the output intensity can vary somewhat inversely with the input intensity. This is not a behavior that one would normally expect for an amplifier, and its occurrence is related to the saturated bidirectional

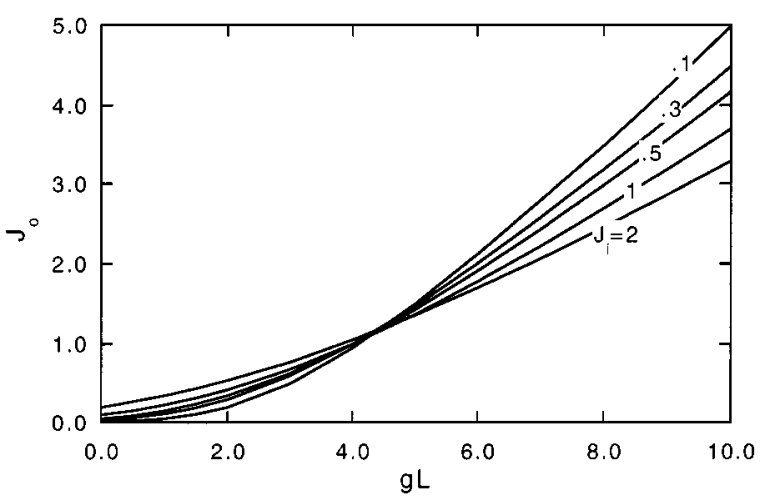

FIG. 4. Normalized output intensity as a function of the gain-length product for various values of the input intensity and a mirror reflection coefficient $R=0.1$. Note that for some values of the gain-length product, the output can vary somewhat inversely with the input intensity. 
nature of these double-pass systems. For a limited range of input intensities, saturation by the right traveling wave can cause a sufficient reduction in the gain for the left traveling wave to produce a decrease in output for an increase in input. Under these conditions a plot of output intensity versus input is analogous to the $I-V$ characteristic of a tunnel diode, and a double-pass amplifier could be an active component in optical circuit design. The very small intersection region of the curves in Fig. 4 is also of interest. It is clear that for the appropriate value of the gain-length product the output intensity is essentially constant as the input intensity is varied by more than a factor of ten. Thus, a double-pass amplifier could find use in optical noise reduction or other optical power-limiting applications.

\section{DISCUSSION}

Because of their advantages of compactness and efficiency, double-pass laser amplifiers are attracting increased attention, and developments in this area have been briefly reviewed here. Analytical solutions have been obtained for the output intensity of double-pass homogeneously broadened high-gain amplifiers having an arbitrary mirror reflectivity. Several graphical representations of these solutions have been described corresponding to a wide range of potentially practical parameter values, and further results can readily be obtained as needed. When suitable limits are taken, these solutions often take on a more explicit form or reduce to previously known results.

The results obtained here are in good qualitative agreement with experimental data. Thus, for example, the curves in Fig. 2 are similar to experimental input-output data obtained with a double-pass YAG master oscillator power amplifier (MOPA) laser system that was developed for use in the Laser Interferometer Gravitational Wave Observatory (LIGO) ${ }^{28}$ This application area has been of particular interest to us, and we have obtained similar experimental results with the double-pass YAG laser amplifier now installed at the LIGO Hanford Observatory.

The implications of these solutions are more general than they might at first appear. While our graphical results haven't focused on frequency effects, the underlying formulas fully include the frequency functions associated with the homogeneously broadened laser medium. Frequencydependent mirror reflectivities, as sometimes employed in practice, can also be readily included. Further generalizations are possible too. For example, some double-pass lasers include intensity-dependent mirror reflectivities, ${ }^{37}$ and it would be straightforward to incorporate any desired intensitydependent reflection function into our analytical formulas.

More substantial generalizations should also be possible when necessary. For example, it has been assumed here that reflecting surfaces only occur on one end of the double-pass amplifier and can be characterized by a simple intensity reflection coefficient. For some applications the amplitude and phase of the output field might also be of interest, and the procedure for conversion from intensity to field variables is discussed in Sec. II. Also, it might sometimes happen that partial reflection occurs at the input side of the amplifier. In this event a use of field rather than intensity reflection coefficients would permit the generalization of our results to describe the regenerative and resonant behavior of a bidirectional amplifier with feedback. For some applications the inclusion of transverse spatial variations of the gain or signal would also be of interest, but this could cause a substantial complication of the laser equations. However, if the profile of the field is not changed significantly by the saturation, the model employed here can readily be generalized to incorporate transverse effects. ${ }^{42}$ While a first integral of such a generalized model would sometimes be possible, it appears that numerical methods would be necessary for a complete solution.

The earlier discussions have implied that our principal interest is in the signal evolution in a double-pass laser amplifier. By changing the sign of the gain coefficient $g$, the same analysis applies to the propagation of an optical field in a saturating double-pass laser absorber. Such a configuration can be taken to represent the pump absorption in an optically-pumped homogeneously-broadened laser amplifier possessing a pump feedback mirror. Several studies of double-pass laser amplifiers have included the possibility of double-passing the pump, and the potential advantages of doing so include increased gain, pumping efficiency, and output power. ${ }^{13-15,20,22,24}$ The formulas developed here would make it possible to calculate the total pump energy absorbed in a double-pass-pumped laser.

\section{ACKNOWLEDGMENTS}

This research was supported in part by the Rall Endowment of Whitman College and by the Summer Undergraduate Research Fellowship (SURF) Program at Caltech. One of the authors (J.M.C.) would also like to express her appreciation to Doug Cook, Rick Savage, Peter King, and others associated with the LIGO program for valuable discussions and technical assistance.

${ }^{1}$ A. M. Prokhorov, Opt. Spektrosk. 14, 73 (1963).

${ }^{2}$ A. Yariv and R. C. C. Leite, J. Appl. Phys. 34, 3410 (1963).

${ }^{3}$ L. W. Casperson, Opt. Commun. 8, 85 (1973).

${ }^{4}$ L. W. Casperson, J. Appl. Phys. 48, 256 (1977), and references therein.

${ }^{5}$ See for example, K. G. Ericson and L. R. Lidholt, Ark. Fys. 34, 557 (1968), and references therein.

${ }^{6}$ D. Grischkowsky, IBM Tech. Discl. Bull. 14, 3485 (1972).

${ }^{7}$ M. B. Klein and R. L. Abrams, IEEE J. Quantum Electron. QE-11, 609 (1975).

${ }^{8}$ D. W. Coutts, W. J. Wadsworth, and E. E. Webb, J. Mod. Opt. 45, 1185 (1998).

${ }^{9}$ A. A. Betin and V. E. Sherstobitov, Bull. Acad. Sci. USSR, Phys. Ser. 47, 105 (1983).

${ }^{10}$ A. A. Betin, N. D. Milovskii, and N. Y. Rusov, Radiophys. Quantum Electron. 28, 873 (1986).

${ }^{11}$ A. A. Betin, N. D. Milovskii, N. F. Rul'kov, and N. Y. Rusov, Radiophys. Quantum Electron. 29, 987 (1986).

${ }^{12}$ I. N. Duling III and R. D. Esman, Electron. Lett. 28, 1126 (1992).

${ }^{13}$ S.Nishi, K. Aida, and K. Nakagawa, Proc. ECOC'90 (1990), Vol. I, pp. 99-102.

${ }^{14}$ V. Lauridsen, R. Tadayoni, A. Bjarklev, J. H. Povlsen, and B. Pedersen, Electron. Lett. 27, 327 (1991).

${ }^{15}$ C. R. Giles, J. Stone, L. W. Stulz, K. Walker, C. A. Burrus, in Optical Amplifiers and Their Applications, 1991 Technical Digest Series, Vol. 13 (Optical Society of America, Washington, D.C., 1991), p. 148.

${ }^{16}$ M. Yamada, M. Shimizu, Y. Ohishi, J. Temmyo, T. Kanamori, and S. Sudo, IEEE Photonics Technol. Lett. 5, 1011 (1993). 
${ }^{17}$ M. Yamada, M. Shimizu, Y. Ohishi, J. Temmyo, T. Kanamori, and S. Sudo, Electron. Lett. 29, 1950 (1993).

${ }^{18}$ E. Delavaque, T. Georges, B. Landousies, and E. Taufflieb, Electron. Lett. 31, 2149 (1995).

${ }^{19}$ R. Lebref, E. Delevaque, B. Landousies, H. Poignant, M. Guibert, and T. Georges, Proceedings of the Topical Meeting on Optical Amplifiers and their Applications (OSA) Vol. 5, Monterey, CA, 11-13 July 1996, pp. $83-86$.

${ }^{20}$ J. J. Pan, Y. Shi, and J. Chen, Proceedings of the 22nd European Conference on Optical Communication (ECOC'96), Oslo, Norway, 15-19 September 1996, pp. 302-303.

${ }^{21}$ T. Miyazaki, K. Inagake, Y. Karasawa, and M. Yoshida, J. Lightwave Technol. 16, 562 (1998).

${ }^{22}$ C. E. Soccolich, V. Mizrahi, T. Erdogan, P. J. LeMaire, and P. Wysocki, OFC'94, Optical Fiber Communication, 1994 Technical Digest Series, Vol. 4, (Optical Society of America, Washington, D.C., 1994), pp. $277-$ 278.

${ }^{23}$ R. Brinkman, I. Baumann, M. Dinand, W. Sohler, and H. Suche, IEEE J. Quantum Electron. 30, 2356 (1994).

${ }^{24}$ I. Baumann et al., IEEE J. Sel. Top. Quantum Electron. 2, 355 (1996).

${ }^{25}$ N. N. Puscas, R. Girardi, D. Scarano, and I. Montrosset, J. Mod. Opt. 45, 847 (1998)

${ }^{26}$ D. P. Shepherd, C. T. A. Brown, T. J. Warburton, D. C. Hanna, and A. C. Tropper, Appl. Phys. Lett. 71, 876 (1997).

${ }^{27}$ U. A. Johann and W. Seelert, Proc. SPIE 1218, 228 (1990).

${ }^{28}$ W. Wiechmann, T. J. Kane, D. Haserot, F. Adams, G. Truong, and J. D.
Kmetec, Technical Digest, Summaries of Papers Presented at the Conference on Lasers and Electro-Optics (CLEO), Vol. 6, San Francisco, CA, 3-8 May 1998.

${ }^{29}$ W. Cunkai and W. Zhiying, Kexue Tongbao 30, 886 (1985).

${ }^{30}$ W. W. Chow and D. Depatie, J. Appl. Phys. 65, 4124 (1989).

${ }^{31}$ D. Huang, D. Liu, S. Yu, M. Zhou, X. Liu, and J. Huang, Electron. Lett. 27, 571 (1991).

${ }^{32}$ G. C. Dente and M. L. Tilton, IEEE J. Quantum Electron. 29, 76 (1993).

${ }^{33}$ L. Goldberg, D. Mehuys, M. R. Surette, and D. C. Hall, IEEE J. Quantum Electron. 29, 2028 (1993)

${ }^{34}$ R. J. Lang, D. Mehuys, D. F. Welch, and L. Goldberg, IEEE J. Quantum Electron. 30, 685 (1994).

${ }^{35}$ E. Gehrig, B. Beier, K.-J. Boller, and R. Wallenstein, Appl. Phys. B: Lasers Opt. 66, 287 (1998).

${ }^{36}$ A. M. Hunter, II and R. O. Hunter, Jr., IEEE J. Quantum Electron. QE-17, 1879 (1981)

${ }^{37}$ A. M. Kamchatnov and A. L. Chernyakov, Sov. J. Quantum Electron. 12, 599 (1982).

${ }^{38}$ A. A. M. Saleh, R. M. Jopson, J. D. Evankow, and J. Aspell, IEEE Photonics Technol. Lett. 2, 714 (1990).

${ }^{39}$ C. R. Giles and E. Desurvire, J. Lightwave Technol. 9, 271 (1991).

${ }^{40}$ M. Azadeh and L. W. Casperson, J. Appl. Phys. 83, 2399 (1998).

${ }^{41}$ H. G. Danielmeyer, J. Appl. Phys. 42, 3125 (1971).

${ }^{42}$ L. W. Casperson, Ph.D. thesis, California Institute of Technology, Pasadena, California, 1971, pp. 311-317 (University Microfilms, Ann Arbor, Michigan, No. 72-469). 